\title{
Saúde materno infantil no Brasil: evolução e programas desenvolvidos pelo Ministério da Saúde
}

\author{
Angélica Capellari Menezes Cassiano \\ Centro Universitário de Maringá (UniCesumar)
}

Edilaine Monique de Souza Carlucci

Centro Universitário de Maringá (UniCesumar)

Cristiane Faccio Gomes

Centro Universitário de Maringá (UniCesumar)

Rose Mari Bennemann

Centro Universitário de Maringá (UniCesumar)

\section{Saúde materno infantil no Brasil: evolução e programas desenvolvidos pelo Ministério da Saúde}

Trata-se de uma revisão de literatura com o objetivo de descrever a evolução da atenção à saúde materno infantil no Brasil desde o Estado Novo até os dias atuais. O levantamento de dados foi realizado em livros, sites do Ministério da Saúde, Legislações do Diário Oficial e artigos das bases de dados Biblioteca Virtual em Saúde (BVS/Bireme), Medline e Scientific Eletronic Library (Scielo). As políticas públicas de saúde materno infantil foram definidas seguindo desde os princípios meramente reprodutivos até aqueles mais amplos, voltados para garantir uma melhor condição de saúde e de vida. Nas últimas décadas, com o auxílio do governo e da sociedade, houve uma progressão no atendimento da saúde da mulher e da criança. No entanto, conclui-se que, apesar da evolução observada, o grande desafio ainda se encontra na redução da morbimortalidade materna, neonatal e infantil.

Palavras-chave: política de saúde, mortalidade infantil, política pública, programa social, saúde

Artigo recebido em agosto de 2013. Versão final em maio de 2014. 
Salud Maternal-Infantil en Brasil: evolución y programas desarrollados por el Ministerio de la Salud

Se trata de una revisión de literatura con el objetivo de describir la evolución de la atención a la salud maternal-infantil en Brasil desde el nuevo estado hasta los días actuales. El levantamiento de los datos fue realizado en libros, sites del Ministerio de la Salud, Legislación del Diario Oficial y artículos de las bases de datos de la Biblioteca Virtual en Salud - BVS/Bireme y la Scientific Eletronic Library - Scielo. Las políticas públicas de salud maternal-infantil fueron definidas desde el principio simplemente reproductivo hasta aquellos más amplio, centrados para garantizar una mejor condición de salud y de vida. En las últimas décadas, con la ayuda del gobierno y de la sociedad, hubo un progreso en el tratamiento de la salud de la mujer y del niño. Sin embargo se concluye que, a pesar de la evolución observada el grande desafío aún se encuentra en la reducción de la mortalidad materna, neonatal e infantil.

Palabras-clave: política de salud, mortalidad infantil, política pública, programa social, salud

\section{Maternal and child health service in Brazil: the evolution of health care and the public policies of the Ministry of Health}

This paper reviews the literature on maternal and child health policies with the objective of describing the evolution of health care for maternal and child health service in Brazil since 1937 to the present day. The data were collected in books, websites of the Ministry of Health, Laws and Articles of the Official Gazette, the Virtual Library data bases in BVS/ Bireme Health, Medline and the Scientific Electronic Library - SciELO. The definition for public policies on maternal and child health ranged from merely reproductive principles to broader ones, in order to ensure better quality of health and life. In recent decades, with the assistance of government and society, there has been advancement in the health care of women and children. However, we conclude that, despite the advancements observed, the greatest challenge still lies in reducing maternal, infant and child mortality rates.

Keywords: health policy, infant mortality, public policy, social program, health 


\section{Introdução}

As políticas públicas de saúde destinadas ao atendimento das necessidades e dos direitos da mulher e da criança são definidas seguindo desde princípios meramente reprodutivos, voltados apenas para a concepção e anticoncepção, até aqueles mais amplos, voltados para garantir uma melhor condição de saúde e de vida (Costa; Bahia; Conti, 2007).

Os movimentos sociais que ocorreram na sociedade brasileira, principalmente o movimento feminista, contribuíram para a construção de novas políticas públicas a partir da década de 1980. Isso resultou na consolidação de leis e programas de saúde que se intensificaram em toda a década de 1990 e se estenderam até os anos 2000, pela formulação e execução de programas e estratégias de saúde pública voltados à atenção materno infantil (SAntos Neto et al., 2008).

A implantação do Programa de Assistência Integral à Saúde da Mulher (PAISM), em 1984, significou uma conquista ao colocar em destaque que a saúde da mulher precisava ser abordada em todos os ciclos da vida e não apenas no período da gravidez. A proposta do programa considerava a necessidade de articulação das ações de pré-natal, assistência ao parto e puerpério, prevenção do câncer e de doenças sexualmente transmissíveis, assistência à adolescente, à menopausa e à anticoncepção (AlmeidA, 2005).

O PAISM foi apenas o início de uma série de mudanças expressivas que ocorreram na reorientação do modelo assistencial médico-privatista vigente. As ações de saúde ainda eram elementares devido à lógica de financiamento do sistema voltada apenas à produtividade dos serviços (SAntos Neto et al., 2008).

No ano de 2000 foi implantado o Programa de Humanização do Pré-Natal e do Nascimento (PHPN), com o principal objetivo de reduzir as altas taxas de morbimortalidade materna, perinatal e neonatal no País (BRASIL, 2005a).

Após a implantação do PHPN, verificou-se que o programa não teve o alcance esperado, pois apresentou dificuldades no acesso aos serviços de saúde, falta de leitos, deficiência de recursos humanos, financeiros e materiais. Mulheres ainda relataram a incerteza de vagas nos hospitais e a preocupação com a peregrinação entre os serviços hospitalares (Dias; Deslandes, 2006; Parada; Tonete, 2008).

$\mathrm{Na}$ busca pela melhoria da assistência materno infantil, o Ministério da Saúde lançou, em 2011, o programa Rede Cegonha. O Rede Cegonha é uma importante estratégia do Governo Federal, uma vez que objetiva a implementação de um novo modelo de atenção à saúde da mulher e da criança, que garanta acesso, acolhimento e resolutividade e reduza, dessa forma, os índices de mortalidade materno infantil (CAVALCANTI, 2010). 
Diante do exposto, este trabalho teve como objetivo descrever a evolução da atenção à saúde materno infantil no Brasil, bem como dos programas desenvolvidos pelo Ministério da Saúde.

\section{Metodologia}

Trata-se de uma revisão de literatura sobre a evolução das ações e programas de atenção à saúde materno infantil no Brasil. O levantamento dos dados foi realizado em livros, sites do Ministério da Saúde, Legislações do Diário Oficial e artigos das bases de dados da Biblioteca Virtual em Saúde (BVS/Bireme), Medline e da Scientific Eletronic Library (Scielo).

A busca foi realizada por meio do método integrado, com utilização dos seguintes descritores: redes de atenção à saúde, atenção primária à saúde, Rede Cegonha, Programa de Assistência Integral à Saúde da Mulher, humanização do parto, humanização do pré-natal e Sisprenatal. Delimitados os descritores, a seleção dos textos foi realizada por leitura exploratória, com o objetivo de conhecer o material, e, posteriormente, por leitura seletiva, na qual foram excluídos estudos regionais. Por fim, a leitura interpretativa, juntamente com a experiência profissional dos pesquisadores, permitiu um maior significado ao estudo.

\section{Evolução da atenção à saúde materno infantil no Brasil}

A história da saúde materno infantil no Brasil foi construída ao longo de várias décadas, recebeu diferentes terminologias e passou por várias gestões. Inicialmente, mulher e criança não ocupavam as posições que atualmente a sociedade e as políticas públicas lhes permitem e garantem (Vıctora et al., 2011).

Entre 1937 e 1945, no governo de Getúlio Vargas, período denominado de Estado Novo, foi implantado o primeiro programa de defesa à saúde materno infantil do Brasil. Suas atividades eram realizadas pelo Departamento Nacional de Saúde do Ministério da Educação e Saúde da Criança (MES), que objetivava apenas normatizar o atendimento ao binômio mãe-filho e combater a mortalidade infantil, sem preocupação com as questões relacionadas à reprodução (BRASIL, 2011d).

Em 1940, Getúlio Vargas transferiu para o Departamento Nacional da Criança (DNCr) as responsabilidades relacionadas à saúde da mãe e da criança, sendo esse, então, o primeiro órgão criado para atender exclusivamente à saúde materno infantil (BRASIL, 2011d).

De acordo com Tyrrell (1997), Getúlio Vargas defendia que a reprodução humana era mais importante para a sociedade do que para o indivíduo, uma vez que o 
motivo principal do Estado e da mulher era melhorar a raça humana com o objetivo de formar homens com condições cada vez melhores para auxiliar no crescimento e desenvolvimento econômico do País. Nesse sentido, toda assistência educacional, sanitária e médica voltada à mulher e à criança tinha por objetivo apenas a busca por uma Nação forte e rica.

Segundo Pereira (1999), para conseguir uma Nação forte e sadia, o DNCr deveria realizar ações para combater a mortalidade infantil, melhorar o pré-natal e voltar a educação para o estímulo a bons hábitos morais e higiênicos. Isso só seria possível por meio do reforço alimentar materno e infantil, do ensino da puericultura às mães, da distribuição de merenda escolar e do ensino de práticas de higiene.

Apesar das ações propostas pelo DNCr, o que se verificou foi a manutenção da pobreza, da exploração excessiva da força de trabalho, grave quadro sanitário e educacional e uma redução menor do que a almejada da mortalidade materno infantil (VIEIRA, 2003). Entretanto, mesmo não obtendo os resultados esperados, pode-se observar redução, mesmo que lenta, da mortalidade infantil ${ }^{1}$, de 168 em 1000 nascidos vivos em 1930, para 150 em 1940 (IBGE, 1999), resultado da implantação de algumas políticas sanitárias nos centros urbanos nacionais.

Nessa época, a maior preocupação do governo era a redução da mortalidade infantil como estratégia para aumentar a população, sem se preocupar com a qualidade da reprodução humana e com as condições de saúde da mulher, que era vista apenas como fonte de reprodução e cuja função era voltada exclusivamente para a criação de braços fortes para servir o País, sem direitos pessoais, sociais e econômicos. Não havia um acompanhamento da sua saúde, nem mesmo durante a gravidez. O governo apenas incentivava a procriação em massa, sem fornecer as condições mínimas necessárias para um bom desenvolvimento gestacional.

Acreditava-se na orientação e responsabilização exclusiva da mãe e da família em relação ao autocuidado e ao cuidado com a criança. A intensificação da melhoria das práticas de higiene e educação sanitária seria suficiente para reduzir os índices de mortalidade infantil. Entretanto a implantação dessas práticas não foi capaz de atingir os objetivos esperados, e a superpopulação, até então estimulada, passou a ser mais um agravante para o precário quadro sanitário e econômico já existente no País.

\footnotetext{
${ }^{1}$ A taxa de mortalidade infantil é calculada da seguinte forma: número de óbitos de menores de um ano de idade, por mil nascidos vivos, em determinado espaço geográfico, no ano considerado (Datasus).
} 
Em 1964, os militares assumiram o governo e, a partir desse momento, a saúde começou a ser vista de maneira individual e não como um fenômeno coletivo e com ações voltadas exclusivamente para a cura (NAgahama; Santiago, 2005).

No que se refere à atenção dada à saúde da mulher e da criança durante o militarismo, na década de 1975, houve a implantação do Programa de Saúde Materno Infantil (PSMI) (BRASIL, 1975). O PSMI tinha dois focos de atuação: o primeiro era a intervenção médica sobre o corpo feminino por meio do uso da cesariana e da esterilização como método contraceptivo preferencial, e o segundo era a redução da morbidade e mortalidade materna e infantil (SANTOS Neto et al., 2008).

De fato, esse programa auxiliou na redução da taxa de mortalidade infantil, que passou de 100 em 1000 nascidos vivos em 1975, para 82,9 em 1980 (IBGE, 1999). No que se refere ao controle da natalidade por meio da esterilização feminina, esse programa também teve sucesso, uma vez que a taxa de fecundidade total apresentou redução. O índice que era de 6,2 filhos para cada mulher, em 1950, caiu, em 1970, para 5,8 e, em 1980, para 4,2 (VIEIRA et al., 2002).

Apesar disso, os programas de saúde passaram a ser fortemente criticados pelo movimento feminista brasileiro, em função do aspecto reducionista que apresentavam. Assim, contribuiu-se para introduzir, na agenda da política nacional, questões como a desigualdade social entre homens e mulheres, sexualidade, dificuldades relacionadas à anticoncepção, doenças sexualmente transmissíveis e a sobrecarga de trabalho doméstico (ÁvILA; BLANDER, 1991).

$\mathrm{Na}$ tentativa de resolver os problemas sanitários e econômicos ocasionados pelo aumento populacional durante o governo anterior, é lançada, como estratégia principal, a esterilização feminina como método anticoncepcional. Nesse período, acreditava-se que a redução do número de habitantes seria a medida mais eficaz para melhorar as condições do País.

A atenção prestada à mulher passa a ter um caráter mais individual do que nas décadas anteriores, porém esse atendimento é fragmentado e restrito apenas ao ciclo gravídico, e a mulher permanece sem atenção durante todas as outras etapas de sua vida. A atenção destinada à criança ainda é voltada exclusivamente para o nascimento, ou seja, o que importava era o fato de ela ter nascido viva ou morta, e, a partir desse momento, ela recebia somente o atendimento curativo.

Foi durante o militarismo que o movimento feminista, impulsionado pela percepção das desigualdades sociais existentes entre os gêneros e pela fragmentação da assistência prestada à população feminina, iniciou a busca por estratégias capazes de mudar o quadro existente na época. As mulheres demonstraram, por meio de movimentos sociais organizados, sua insatisfação quanto à violência corporal sofrida em relação ao uso exagerado de cesarianas e 
esterilizações. Difundiu-se o entendimento de que a prática de castração era apenas uma tentativa ineficaz de solucionar os problemas sociais existentes.

Diante dessas argumentações, em 1983, foi elaborado, pelo Ministério da Saúde, o Programa de Assistência Integral à Saúde da Mulher (PAISM), com a publicação do documento "Assistência Integral à Saúde da Mulher: bases de ação programáticas", em 1984. Esse programa foi uma das maiores conquistas do movimento feminista, resultante da mobilização das mulheres brasileiras em busca da cidadania, dos direitos e do acesso ao espaço público (BRASIL, 1983).

O PAISM foi o primeiro programa lançado pelo Governo Federal com o propósito de planejamento familiar no Brasil (BRASIL, 2011c). Tem seus princípios e diretrizes voltados para a orientação do atendimento integral à saúde das mulheres e inclui, em suas ações, atividades educativas, preventivas, de diagnóstico, tratamento e recuperação da saúde. Atende às necessidades clínico-ginecológicas, pré-natal, parto, puerpério, climatério, doenças sexualmente transmissíveis, câncer de colo de útero e mama (BRASIL, 1983; OsIs, 1998).

No âmbito das relações de mercado, o que mais contribuiu para proteger a saúde da mulher e da criança foi a Constituição Federal de 1988, que, em alguns de seus artigos, descreve os direitos reprodutivos adquiridos. Entre eles, destacam-se:

[...] o direito das presidiárias de permanecerem com seus filhos durante o período de amamentação; a proteção à maternidade e à infância; a licença à gestante sem prejuízo do emprego e do salário, com a duração de cento e vinte dias; a licença-paternidade nos termos fixados em lei; a assistência gratuita aos filhos e dependentes, desde o nascimento até seis anos de idade em creches e pré-escolas; a proteção especialmente à gestante e ao planejamento familiar (BRASIL, 1988) .

Apesar de o pré-natal estar presente no topo de todas as ações relacionadas à assistência da mulher, alguns problemas ainda ficaram pendentes, como o acesso ao serviço médico em algumas regiões do País, a qualidade da atenção, o vínculo entre o pré-natal e o parto, a humanização da atenção, a elevada taxa de mortalidade materna e altos índices de esterilização (COSTA, 1999).

O PAISM foi um dos maiores avanços na atenção à saúde da mulher e da criança. Constituiu um marco histórico de extrema importância para o Brasil, pois é a primeira vez que o governo deixa de pensar somente no tratamento individual e na cura de doenças e inclui, em seu programa, questões referentes à família. A esterilização deixou de ser o método contraceptivo mais usado e se passou a destacar a importância do planejamento familiar e de ações educativas de 
prevenção à gravidez indesejada e doenças sexualmente transmissíveis. A relevância desse programa se torna ainda maior quando se destaca a proposta social de mudança para a integralidade da atenção à saúde da mulher, que passa a incluir a prevenção e o tratamento de agravos à saúde desde a adolescência até a terceira idade e não apenas durante o período gestacional.

Mesmo com a redução da taxa de mortalidade materna ${ }^{2}$ de 68,9 óbitos para cada 100 mil nascidos vivos em 1980 para 45 óbitos em 1996 (RIPSA, 2004)³ , o Brasil ainda permanecia com índices altíssimos de mortalidade materna quando comparado com os países desenvolvidos, uma vez que, nesses países, ocorriam menos de 10 mortes para cada 100 mil nascidos vivos.

Segundo Tyrrell (1997), após três décadas da implantação do PAISM, as condições das mulheres e crianças do nosso país ainda eram gravíssimas, tendo em vista a persistência dos altos índices de morbimortalidade materna e infantil.

Serruya, Cecatti e Lago (2004) relatam que essa política foi bem estruturada, mas não foi efetivamente implantada em todo o território nacional, e, apesar de proporcionar avanço expressivo dos direitos reprodutivos das mulheres brasileiras, sua implantação sofreu dificuldades políticas, financeiras e operacionais, o que impediu que o programa se transformasse, de fato, em agente transformador da saúde das mulheres.

Diante disso, em junho de 2000, foi lançado o Programa de Humanização no Pré-natal e Nascimento (PHPN), pela Portaria/GM no 569, que teve como elementos estruturadores a humanização e o respeito aos direitos reprodutivos (BRASIL, 2000a).

O PHPN foi implantado com o objetivo principal de reduzir a morbimortalidade materna e neonatal, proporcionar atendimento humanizado e melhorar o acesso, a cobertura e a qualidade do acompanhamento do pré-natal, da assistência ao parto e puerpério das gestantes e do recém-nascido (Santos Neto et al., 2008).

A atenção pré-natal e puerperal qualificada e humanizada implica a realização de condutas acolhedoras e sem intervenções desnecessárias; o acesso facilitado aos serviços de saúde que tenham ações que integrem todos os níveis da atenção;

\footnotetext{
${ }^{2}$ A taxa de mortalidade materna é calculada da seguinte forma: número de óbitos femininos por causas maternas, por 100 mil nascidos vivos, em determinado espaço geográfico, no ano considerado (Datasus).

${ }^{3}$ Os dados da RIPSA são oficiais, ou seja, não corrigidos, o que pode ocasionar divergência com dados de outras fontes.
} 
e a promoção, prevenção e assistência à saúde da gestante e do recém-nascido, desde $o$ atendimento ambulatorial até o atendimento hospitalar para alto risco (BRASIL, 2005a). Além disso, promove o encorajamento de vínculos afetivos entre mãe e filho logo após o nascimento, e garante uma assistência segura, confiável e suave pelos profissionais durante todo o tempo em que ambos permanecerem na maternidade (Silva; Christoffel; SouzA, 2005).

Entre as conquistas adotadas na humanização do parto e nascimento, está a Lei $\mathrm{n}^{\circ}$ 11.108, de abril de 2005, que garante à parturiente um acompanhante durante o trabalho de parto, parto e pós-parto imediato, no âmbito do Sistema Único de Saúde - SUS (BRASIL, 2005b). A participação do acompanhante durante o processo de parturição envolve o apoio emocional, importante na medida em que compartilhar o momento do parto e nascimento com o acompanhante pode ser um facilitador do trabalho de parto para a parturiente.

Com a implantação do PHPN, o atendimento destinado à saúde da mulher passa a ter um enfoque diferenciado. Inicia-se uma preocupação com a qualidade do atendimento, estabelecendo a humanização como um dos princípios norteadores da assistência. A gestante, que até esse momento não tinha conhecimento em relação ao lugar em que daria à luz, tem agora o direito de saber qual é a maternidade de referência. Isso lhe dá maior segurança durante todo o período gestacional, uma vez que ela sabe qual instituição deve procurar caso necessite de atendimento. Ela passa a ter o direito de um acompanhante durante o pré-parto, parto e puerpério imediato. Isso proporciona à mulher maior conforto e segurança durante todo período de internamento. Outra novidade estabelecida pelo programa é o alojamento conjunto, ou seja, todo bebê que nascer com boas condições de saúde deve permanecer em contato com a mãe, durante todo o tempo e não somente durante a amamentação.

Para incentivar a implementação do PHPN pelos Municípios, o Governo Federal estabeleceu, dentro do próprio programa, incentivo financeiro de $\mathrm{R} \$ 10$ para cada atendimento inicial à gestante, e de $\mathrm{R} \$ \mathbf{4 0}$ na conclusão do pré-natal, porém esses valores só seriam repassados se os critérios mínimos estabelecidos pelo programa fossem cumpridos. Esses critérios são:

Realizar a primeira consulta de pré-natal até o quarto mês de gestação; garantir a realização dos seguintes procedimentos: no mínimo, seis consultas de pré-natal, preferencialmente uma no primeiro trimestre, duas no segundo trimestre e três no terceiro trimestre da gestação; uma consulta no puerpério, até 42 dias após o nascimento; exames laboratoriais: a) ABO-Rh, na primeira consulta; b) VDRL, um exame na primeira consulta e outro próximo à trigésima semana da gestação; c) Urina rotina, um exame na primeira consulta e outro próximo à trigésima semana da gestação [...] (BRASIL, 2000b, p. 6-7). 
Para acompanhar, planejar e avaliar a qualidade do pré-natal e puerpério das mulheres inseridas no PHPN e monitorar o pagamento do incentivo financeiro, foi criado pelo Datasus um software denominado SISPRENATAL (Sistema Eletrônico para Coleta de Informações sobre o Acompanhamento das Gestantes Atendidas no SUS).

O SISPRENATAL foi desenvolvido para permitir o acompanhamento da gestante desde o início da gravidez até o puerpério. Cada município fica responsável por inserir os dados no programa e, se o procedimento é realizado de forma correta, é possível obter uma visão geral das condições de saúde da gestante e não apenas o número absoluto das consultas realizadas. Com a criação do SISPRENATAL foi possível fazer o monitoramento das gestantes em cada região do Brasil, o que facilitou o repasse financeiro de acordo com a necessidade de cada região.

A análise do PHPN, após uma década do lançamento, mostrou que o programa não teve a abrangência esperada, e manteve, em todo território brasileiro, cumprimento inferior das metas estabelecidas e um modelo biomédico de assistência (Trevisan, 2002; Coimbra, 2005; Silva; Cecatti; Serruya, 2005).

Em 1995, foram registradas 1,2 consultas de pré-natal para cada parto realizado no SUS. Em dezembro de 2002, essa razão era de 4,4 consultas de pré-natal para cada parto. Mesmo com o aumento do número de consultas de pré-natal, a qualidade dessa assistência ainda permanecia ruim (BRASIL, 2011c).

As propostas estabelecidas pelo PHPN, de fato, parecia que seriam, por si sós, capazes de modificar o grave quadro de saúde da mulher e da criança brasileira e reduzir os altos índices de morbimortalidade materna e neonatal, mas, infelizmente, o que se observou foi apenas um aumento no número das consultas de pré-natal e não uma melhora significativa da qualidade da assistência. $A$ humanização do atendimento ficou restrita aos grandes centros urbanos, permanecendo a maior parte da população feminina à mercê de um atendimento pouco resolutivo, limitado e pouco humanizado.

Com o reconhecimento de que a redução da mortalidade ainda era um desafio para o Brasil, em março de 2004 foi lançado, pela Presidência da República, o Pacto Nacional pela Redução da Mortalidade Materna e Neonatal, que tem como meta reduzir essas mortes em $5 \%$ ao ano. Seus princípios estão voltados para o respeito aos direitos humanos de mulheres e crianças; a consideração das questões de gênero, dos aspectos étnicos e raciais e das desigualdades sociais e regionais; a decisão política de investimentos na melhoria da atenção obstétrica e neonatal; e a ampla mobilização e participação de gestores e organizações sociais (BRASIL, 2004).

Outra preocupação do pacto é a alta taxa de cesarianas, que pode ser um motivo de complicações no parto. A cesariana representa $80 \%$ dos partos realizados via 
planos privados de saúde e $26 \%$ no sistema público, o que equivale a uma taxa média de 40\%. Essa situação coloca o País entre os líderes mundiais em frequência de cesariana (BRASIL, 2006). Essa intervenção, realizada de forma desnecessária, pode acarretar riscos de complicações como infecções, hemorragias e complicações anestésicas que contribuem para o aumento das taxas de mortalidade materna e podem gerar também complicações para o recém-nascido.

A cesariana, que teve sua prática estimulada em governos anteriores, tornou-se um grande problema de saúde pública e passou a ser desencorajada, dando prioridade para políticas de incentivo ao parto normal, para tentar corrigir os prejuízos causados pela epidemia de cesarianas. O Brasil ainda sofre as consequências causadas pelo incentivo a essa prática e tenta, através de novas políticas públicas, incentivar o parto natural, uma vez que, com as altas taxas de cesariana e de mortalidade materna e neonatal, pode-se sugerir que ambas estejam associadas, devido aos diversos riscos que essa prática cirúrgica oferece para a gestante e o bebê.

Nas últimas décadas, com o auxílio do governo e da sociedade, o Brasil progrediu muito no atendimento à mulher e à criança. Dados do Ministério da Saúde apontam que, entre o período de 1990 e 2010, o Brasil reduziu a Razão de Mortalidade Materna (RMM) de 141 para 68 óbitos maternos por 100 mil nascidos vivos, o que representa uma redução de 51\% (BRASIL, 2011c). Em relação à mortalidade infantil, houve decréscimo de cerca de $40 \%$ desses óbitos nas últimas duas décadas. Em 1990, a mortalidade infantil era de 26,6 óbitos por 1000 nascidos vivos, e em 2010, de 16,2 óbitos por 1000 nascidos vivos. Apesar do esforço, os níveis da mortalidade permanecem elevados quando comparados com os níveis de outros países com semelhantes índices de desenvolvimento econômico (BRASIL, 2011d).

Como a morbimortalidade é um processo complexo e multifatorial, o Brasil ainda enfrenta muitos desafios para sua redução, entre eles, melhorar a qualidade do pré-natal e das ações de educação em saúde, permitir o acesso, no pré-natal, aos exames necessários, favorecer o conhecimento prévio da gestante do local do parto, fortalecer o atendimento à mulher e à criança no puerpério e o acompanhamento do desenvolvimento da criança (CAVAlCANTI, 2010; BRASIL, 2011a).

É com o objetivo de vencer esses desafios e melhorar cada vez mais o atendimento à gestante e à criança, priorizando a atenção continuada à saúde, que o governo vem desenvolvendo novas estratégias de ações em relação à saúde materno infantil.

\section{Redes de Atenção à Saúde: conceito e definições}

Como estratégia para superar a fragmentação da atenção à saúde e aprimorar o funcionamento político-institucional do SUS, o Ministério da Saúde, o Conselho 
Nacional de Secretários da Saúde (CONASS) e o Conselho Nacional de Secretarias Municipais de Saúde (CONASEMS), durante a reunião da Comissão Intergestores Tripartite, instituíram o posicionamento sobre as Redes de Atenção à Saúde. Posicionamento esse que está materializado na Portaria 4.279, de 30 de dezembro de 2010, pelas diretrizes para a organização da Rede de Atenção à Saúde no âmbito do SUS (BRASIL, 2010).

O planejamento político de redes é uma alternativa cada vez mais presente e está associada a maior flexibilidade, integração e interdependência entre organizações estatais, sociais e empresariais, constituindo saída para a crise contemporânea dos sistemas de atenção à saúde (FleURY; OUVERNEY, 2007).

As redes de atenção à saúde têm como objetivo garantir a integralidade da atenção à saúde, a partir do gerenciamento de pontos de atenção e diagnóstico, com diferentes níveis de complexidade distribuídos em um território (PINHEIRO FILHO; SARTI, 2012). A integração dos cuidados consiste na coordenação durável das práticas clínicas destinadas a alguém que sofre com problemas de saúde e assegura a continuidade dos diferentes serviços requeridos (HARTZ; ContANDRIOPOULOS, 2004).

\section{Rede Cegonha}

A partir da perspectiva de promover melhoria na assistência à saúde materno infantil, em 2011, o Ministério da Saúde lançou a Rede Cegonha, programa que foi normatizado pela Portaria no 1.459, de 24 de junho de 2011. Essa estratégia tem como objetivo aplicar um novo modelo de atenção à saúde da mulher e da criança, com foco no parto, nascimento, crescimento e desenvolvimento da criança, garantia de acesso, acolhimento e resolutividade e a redução da taxa de mortalidade materna e neonatal (BRASIL, 2011e).

A Rede Cegonha tem como princípios o respeito, a proteção e a realização dos direitos humanos; o respeito à diversidade cultural, étnica e racial; o enfoque de gênero; a promoção da equidade; a participação e mobilização social; a garantia dos direitos sexuais e reprodutivos de mulheres, homens, jovens e adolescentes; e a compatibilização com as atividades das redes de atenção à saúde materna e infantil em desenvolvimento nos Estados (BrasIL, 2011f). Prevê ações para a ampliação e melhoria do acesso e da qualidade da assistência à mulher e à criança por meio da vinculação da gestante à unidade de referência para assistência ao parto e transporte, da realização de boas práticas de atenção ao parto e nascimento seguro e da atenção à saúde da criança de zero a 24 meses com qualidade e resolutividade. Essas ações estão inseridas em quatro componentes estruturantes da estratégia: Pré-Natal; Parto e Nascimento; 
Puerpério e Atenção integral à Saúde da Criança; e Sistema Logístico, Transporte Sanitário e Regulação (BrasIL, 2011e).

Cada um desses componentes compreende uma série de ações de atenção à saúde, entre elas: oferecer o teste de HIV, sífilis e teste rápido de gravidez; orientar e ofertar métodos contraceptivos; fornecer o mínimo de seis consultas de pré-natal durante a gestação, exames clínicos e laboratoriais; garantir o direito de leito e de vinculação da gestante a uma determinada maternidade ou hospital público e valetransporte ou vale-táxi até o local no dia do parto; qualificar os profissionais de saúde; criar centros de gestante e do bebê para a assistência à gravidez de alto risco; permitir o acesso ao pré-natal de alto risco em tempo adequado; oferecer o Samu Cegonha ao recém-nascido que necessite de transporte de emergência com ambulâncias equipadas com incubadoras, ventiladores neonatais; e promover ações de incentivo ao aleitamento materno (CARNEIRO, 2013).

A proposta do governo é disponibilizar 9,4 bilhões de reais para amparar os dois milhões de gestantes atendidas pelo SUS. Esse valor será aplicado na construção de uma rede de cuidados primários para a saúde da mulher e de crianças, com início do atendimento pela Amazônia e pela região Nordeste, locais que registram as maiores taxas de mortalidade materna e infantil no País (BRASIL, 2011g).

A Rede Cegonha é uma proposta do Governo Federal, mas caberá aos Estados e Municípios a sua aplicação. Até março de 2012, 24 estados haviam aderido ao programa (BRASIL, 2012a). Em setembro desse mesmo ano, o Ministério da Saúde divulgou que os estados do Rio Grande do Norte, Acre, Pernambuco e Mato Grosso seriam beneficiados com $\mathrm{R} \$ \mathbf{9 2 , 9}$ milhões de recursos para qualificar e ampliar a assistência à gestante e ao bebê com a estratégia da Rede Cegonha. O Estado do Rio Grande do Norte terá R\$ 46,8 milhões; o Acre, R\$ 18,6 milhões; Pernambuco, $\mathrm{R} \$$ 8,5 milhões; e Mato Grosso, R\$ 19 milhões (BrasIL, 2012b).

Durante a 2a Reunião do Comitê de Mobilização Social, o Ministério da Saúde afirmou que, após um ano da fundação da Rede Cegonha, foram realizadas mais de 20 milhões de consultas para o pré-natal. São 133\% a mais que os 8,6 milhões registrados em 2003. Isso significa a criação de ações qualificadas desde o prénatal até a atenção ao parto, o nascimento, o resguardo e o desenvolvimento da criança até dois anos (BRASIL, 2012a).

Outra conquista obtida através da estratégia Rede Cegonha é a ampliação dos exames do teste do pezinho. Assim, a intenção é aumentar e qualificar os serviços e incluir, gradativamente, a ampliação do acesso aos exames, tratamentos e acompanhamentos de todas as doenças definidas no escopo do Programa Nacional de Triagem Neonatal - PNTN (BRASIL, 2012c). 
A qualificação profissional também faz parte da estratégia da Rede Cegonha, pois os Ministérios da Saúde e da Educação lançaram o Programa Nacional de Residência em Enfermagem Obstetrícia (PRONAENF).

O programa planeja capacitar enfermeiros e obstetras para serem inseridos no SUS, nas regiões que aderiram à estratégia Rede Cegonha. O programa Pronaenf é uma ação que contribui com a Rede Cegonha, qualifica os enfermeiros para prestar um serviço humanizado e de qualidade para a criança e para mulher e capacita os profissionais a atuarem em diferentes momentos: pré-natal, parto, nascimento e pós-parto (BRASIL, 2012d).

A Rede Cegonha é, até então, o programa mais completo já elaborado pelo Governo Federal. Suas ações são voltadas para todas as etapas da vida da mulher e abrange estratégias que vão desde orientação em relação ao cuidado com o corpo, com o uso de métodos contraceptivos, atendimento da gestante, puérpera e recémnascido, até ações voltadas ao atendimento da criança até dois anos de idade.

Consolida-se, aqui, a assistência ao parto humanizado e a capacitação de profissionais para exercerem suas funções de forma humanizada e eficiente. Outra grande conquista é a presença do pai durante todo o período gravídico, proporcionando confiança e segurança à gestante. No entanto, mesmo diante dos grandes avanços decorridos da criação da Rede Cegonha, percebe-se que esse programa pode ser considerado como uma junção dos programas anteriormente implantados acrescido de ações para o atendimento das necessidades atuais.

\section{Considerações finais}

Desde o início do século XX, a saúde materno infantil esteve presente nas ações de saúde pública com a implantação de diversos programas, que foram se consolidando ao longo dos anos. Esses programas apresentaram estratégias e evoluções, surgidas de acordo com as necessidades da época, no entanto, nem todas as ações foram positivas para o cenário materno infantil. Entre elas podemos destacar o incentivo à cesariana, procedimento que até hoje permanece com taxas muito altas em todo país e que contribui de forma negativa para a saúde da mãe e da criança.

É importante ressaltar que todos os programas já elaborados possuem um objetivo em comum; diminuir as taxas de morbimortalidade materna e infantil, com ações voltadas para esse propósito. De fato, houve redução nessas taxas, entretanto os índices ainda permanecem altos.

Diante dessas observações, levanta-se a hipótese da necessidade de real efetivação dessas políticas e do acompanhamento da qualidade do serviço 
prestado. Mesmo com o grande avanço observado nos programas da última década e com a ampliação no número de atendimentos e consultas, não se pode afirmar que a qualidade dos serviços prestados é a mesma que a almejada. Dessa forma, sugere-se que estratégias voltadas para o acompanhamento da qualidade dos serviços sejam formuladas, para que se tenha o máximo possível de certeza de que as políticas elaboradas estão de fato sendo implantadas de forma eficiente.

\section{Referências bibliográficas}

AlmeIDA, C. M. O movimento da reforma sanitária: uma visão crítica. In: Congresso Nacional Da Rede Unida - 20 anos de parcerias na saúde e na educação, 6, 2005. Belo Horizonte. Anais. VI Congresso da Rede UNIDA, Belo Horizonte: Rede UNIDA, 2005. p. 25-32.

Ávila, M. B. E.; Bandler, R. A Contracepção no Brasil 1980-1990. Recife: SOS Corpo, 1991. [Mimeo].

BrasIL. Ministério da Saúde. Secretaria Nacional de Saúde. Coordenação de Proteção Materno infantil. Programa nacional de saúde materno infantil. Brasília: Ministério da Saúde, 1975.

Ministério da Saúde. Programa de assistência integral à saúde da mulher. Brasília: Ministério da Saúde, 1983.

Constituição Federal (1988). Constituição da República Federativa do Brasil. Brasília, Senado Federal: Centro Gráfico, 1988.p. 292.

Ministério da Saúde. Secretaria da Saúde. Portaria n 569, de 1 de julho de 2000. Institui o programa de humanização no pré-natal e nascimento, no âmbito do sistema único de saúde. Diário Oficial da União; Poder Executivo. Brasília: Ministério da Saúde, 2000a.

- Ministério da Saúde. Programa de humanização no pré-natal e nascimento:informações para gestores e técnicos. Brasília: Ministério da Saúde, 2000b.

- Ministério da Saúde. Secretaria de Atenção à Saúde. Departamento de Ações Programáticas Estratégicas. Pacto para a redução da mortalidade materna e neonatal. Brasília: Ministério da Saúde, 2004.

- Ministério da Saúde. Secretaria de Atenção à Saúde. Departamento de AçõesProgramáticas Estratégicas. Pré-natal e puerpério: atenção qualificada e humanizada: manual técnico. Brasília: Ministério da Saúde, 2005a.

. Ministério da Saúde. Secretaria da Saúde. Lei n 11.108, de 7 de abril de 2005. Garantir as parturientes o direito á presença de acompanhante durante o trabalho de parto, parto e pós-parto imediato, no âmbito do sistema Único de Saúde. Diário Oficial da União; Poder Executivo, 2005b. 
Ministério da Saúde. Agencia Nacional de Saúde Suplementar. Parto normal versus cesariana: o papel do Estado e das agencias reguladoras. Brasília: Ministério da Saúde, 2006.

Ministério da Saúde. Secretaria da Saúde. Portaria no 4.279, de 30 de dezembro de 2010. Estabelece as diretrizes para a organização da Rede de Atenção à Saúde no âmbito do Sistema Único de Saúde (SUS). Diário Oficial da União; Poder Executivo, 2010.

Ministério da Saúde. Secretaria de Atenção à Saúde. Área Técnica de Saúde da Criança e Aleitamento Materno. Gestões e gestores de políticas públicas de atenção à saúde da criança: 70 anos de história. Brasília: Ministério da Saúde, 2011a.

Ministério da Saúde. Secretaria de Atenção à Saúde. Departamento de Ações Programáticas Estratégicas. Política Nacional de Atenção Integral a Saúde da Mulher Princípios e Diretrizes. Brasília: Ministério da Saúde, 2011b.

. Ministério da Saúde. Secretaria de Vigilância em Saúde. Saúde Brasil 2011: uma análise da situação de saúde e a vigilância da saúde da mulher. Brasília: Ministério da Saúde, 2011c.

- Ministério da Saúde. Secretaria de Vigilância em Saúde. Saúde Brasil 2011: uma análise da situação e de evidências selecionadas de impacto de ações de vigilância em saúde. Brasília: Ministério da Saúde, 2011d.

. Ministério da Saúde. Secretaria da Saúde. Portaria n 1.1459, de 24 de junho de 2011. Institui, no âmbito do Sistema Único de Saúde - SUS - a Rede Cegonha. Diário Oficial da União; Poder Executivo, 2011e.

- Ministério da Saúde. Secretaria de Atenção à Saúde. Manual prático para implementação da Rede Cegonha. Brasília: Ministério da Saúde, $2011 \mathrm{f}$.

- Ministério da Saúde. Secretaria de Atenção a Saúde. Presidenta Dilma lança Rede Cegonha, com investimentos de $R \$ \$$ 9,4 bilhões para atendimento a mães e bebês. 2011g. Disponível em http://www2.planalto.gov.br/imprensa/ releases/presidenta-dilma-lanca-rede-cegonha-com-investimentos-de-r-9-4bilhoes-para-atendimento-a-ma es-e-bebes. Acesso em: 27 de maio de 2013.

. Ministério da Saúde. Il Reunião do comitê de mobilização social da rede cegonha. 2012 a. Disponível em http://portal.saude.gov.br/ portal/arquivos/ pdf/ BALANCO_REDECEGONHA_MARCO2012.PDF. Acesso em 24 de maio de 2013. . Ministério da Saúde. Secretaria de Atenção a Saúde. Mais de $R \$$ 92,9 milhões da rede cegonha para quatro estados. 2012b. Disponível em: http://www2. planalto. gov.br/imprensa/noticias-de-governo/mais-r-92-9-mi-da-rede-ce gonha-para-quatro-estados. Acesso em: 27 de maio de 2013.

Ministério da Saúde. Secretaria da Saúde. Rede Cegonha amplia

exames do teste do pezinho. 2012c. Disponível em: http:// portalsaude.saude.gov.br/portalsaude /noticia/8402/162/rede-cegonha-amplia\%3Cbr\%3Eexames-do-teste-do-pezinho.html. Acesso em 24 de maio de 2013.

Ministério da Saúde. Enfermeiros obstetras serão capacitados para assistência às mães e bebês. 2012d. Disponível em: http://www.brasil.gov.br/ 
noticias/arquivos/2012/09/10/rede-cegonha-capacitara-enfermeiros-obstetras. Acesso em 23 de maio de 2013.

CARneiro, R. G. Dilemas antropológicos de uma agenda de saúde pública: Programa Rede Cegonha, pessoalidade e pluralidade. Interface-Comunicação, Saúde, Educação, v.17, n.44, p.49-59, 2013.

Cavalcanti, P. C. S. O modelo lógico da Rede Cegonha. 2010. 25 f. Monografia (Especialista) - Departamento de Saúde Coletiva do Centro de Pesquisas Aggeu Magalhães, Fundação Oswaldo Cruz, Recife, 2010.

CoImbra, L.C. et al. Inadequate utilization of prenatal care in two Brazilian birth cohorts. Brazilian Journal Of Medical And Biological Research, v. 9, n. 40, p.1195$1202,2005$.

CostA, A.M. Desenvolvimento e implantação do PAISM no Brasil. In: GIFFIN, K.; CostA, S. H. (orgs.). Questões da saúde reprodutiva. Rio de Janeiro: Fiocruz,1999, p. 319-335.

Costa, A. M.; BAhIA, L.; Conte, D. A saúde da mulher e o SUS: laços e diversidades no processo de formulação, implantação e avaliação das políticas de saúde para mulheres do Brasil. Saúde em Debate, Rio de Janeiro, v. 31, n. 75/76/77, p.13-24, dez. 2007.

DiAs, M. A. B.; DesLANDES, S. F. Expectativas sobre a assistência ao parto de mulheres usuárias de uma maternidade pública do Rio de Janeiro, Brasil: os desafios de uma política pública de humanização da assistência. Caderno de Saúde Pública, v. 22, n. 12, p. 2647-2655, 2006.

Ferreira, C. E. C. Avaliação da mortalidade materna a partir de dados censitários para o Brasil e regiões em 1980. Disponível em: http://www.abep.nepo. unicamp.br/docs/anais/pdf/1988/T88V04A24.pdf. Acesso em: 30 de maio de 2013. Fleury, S.; Ouverney, A. M. Gestão de redes: a estratégia de regionalização da política de saúde. Rio de Janeiro: Editora FGV, 2007.

Hartz, Z. M. A.; Contandriopoulos, A. P. Integralidade da atenção e integração de serviços de saúde: desafios para avaliar a implantação de um "sistema sem muros". Caderno de Saúde Pública, v. 20, n. 2, p. 331-336, 2004.

IBGE. Instituto Brasileiro de Geografia e Estatística. Evolução e perspectiva da mortalidade infantil no Brasil. Estudos e pesquisas informação demográfica e socioeconômica, v. 1, n. 2, p. 19-22, 1999.

Nagahama, E.; Ssantiago, S. M. A institucionalização médica do parto no Brasil. Ciência \& Saúde Coletiva, v. 10, n. 3, p. 651-657, 2005.

Osıs, M. J. M. D. Paism: um marco na abordagem da saúde reprodutiva no Brasil. Caderno de Saúde Pública, v.14, supl. 1, p. 25-32, 1998.

Pereira, A. R. A criança no Estado Novo: uma leitura na longa duração. Revista Brasileira de História, v. 19, n. 38, p.165-198, 1999.

Pinheiro Filho, F. P.; Sarti, F. M. Falhas de mercado e redes em políticas públicas: desafios e possibilidades ao Sistema Único de Saúde. Ciência \& Saúde Coletiva, v. 17, n. 11, p. 2981-2990, 2012. 
RIPSA. Rede Interagencial de Informações para Saúde. Indicadores e dados básicos. Brasil, 2004. Disponível em: www.datasus.gov.br. Acesso em: 30 de maio de 2013. Santos Neto, E. T. et al. Políticas de Saúde Materna no Brasil: os nexos com indicadores de saúde materno infantil. Saúde Sociedade, v.17, n. 2, p.107-119, 2008.

Serruya, S. J.; Cecattı, J. G.; Lago, T. D. G. O programa de Humanização do Parto e Nascimento do Ministério da Saúde. Caderno de Saúde Pública, v. 5, n. 20, p.12811289, 20 maio, 2004.

Silva, J. L. P.; Cecattı, J. G.; Serruya, S. J. A qualidade do pré-natal no Brasil. Revista Brasileira de Ginecologia e Obstetrícia, v. 27, n. 3, p.103-105, 2005.

Silva, L. R.; Christoffel, M.; SouzA, K. V. História, conquistas e perspectivas no cuidado à mulher e à criança. Texto Contexto Enfermagem, v. 4, n. 14, p. 585-593, dez., 2005.

TREVISAN, M. R.et al.Perfil da assistência pré-natal entre usuárias do Sistema Único de Saúde em Caxias do Sul. Revista Brasileira de Ginecologia e Obstetrícia, v. 24, n. 5, p. 293-299, jun., 2002.

TyrrelL, M. A. R. Programas nacionais de saúde materno infantil: impacto social e inserção da enfermagem. Ana Nery Revista de Enfermagem, v. 8, n. 1, p.119-134, jul., 1997.

VIEIRA, G. F. A ação do Departamento Nacional da Criança no Estado Novo: Educação, Saúde e Assistência. 2003. Dissertação (Mestrado) - UFRJ, Rio de Janeiro.

VIEIRA, E. M. et al. Características do uso de métodos anticoncepcionais no Estado de São Paulo. Revista de Saúde Pública, v. 36, n. 3, p. 263-270, 2002.

Victora, C. et al Saúde de mães e crianças no Brasil: progressos e desafios. The Lancet, v. 2, n. 1, p. 32-46, maio, 2011.

Angélica Capellari Menezes Cassiano

Mestranda em Promoção da Saúde pelo Centro Universitário de Maringá, Paraná (UniCesumar). Contato: angelicacapellari@hotmail.com

Edilaine Monique de Souza Carlucci

Mestranda em Promoção da Saúde pelo Centro Universitário de Maringá, Paraná (UniCesumar). Contato: edilaine_carlucci@hotmail.com

Cristiane Faccio Gomes

Pós-Doutora em Saúde Coletiva pela Universidade Estadual de Londrina (UEL), professora e orientadora do programa de mestrado em Promoção da Saúde do Centro Universitário de Maringá, Paraná (UniCesumar). Contato: crisgomes@cesumar.br

Rose Mari Bennemann

Doutora em Saúde Pública pela Universidade de São Paulo (USP), professora e orientadora do programa de mestrado em Promoção da Saúde do Centro Universitário de Maringá, Paraná (UniCesumar). Contato: rosemari@cesumar.br 